\title{
Potential impact of host immunity on malaria treatment outcome in Tanzanian children infected with Plasmodium falciparum Anders Enevold*1, Watoky MMM Nkya2 ${ }^{2}$, Michael Theisen ${ }^{3}$, Lasse S Vestergaard ${ }^{1,4}$, Anja TR Jensen ${ }^{1}$, Trine Staalsoe ${ }^{1}$, Thor G Theander ${ }^{1}$, Ib C Bygbjerg ${ }^{1}$ and Michael Alifrangis ${ }^{1}$
}

\author{
Address: ${ }^{1}$ Centre for Medical Parasitology, Institute of International Health, Immunology and Microbiology, University of Copenhagen, \\ Copenhagen, Denmark, ${ }^{2}$ Mbeya Referral Hospital (MRH), Mbeya, Tanzania, ${ }^{3}$ Department of Infectious Disease Immunology, Statens Serum \\ Institute, Copenhagen, Denmark and ${ }^{4}$ Department of Epidemiology, Statens Serum Institute, Copenhagen, Denmark \\ Email: Anders Enevold* - enevold@cmp.dk; Watoky MMM Nkya - mmmnkya@yahoo.com; Michael Theisen - mth@ssi.dk; \\ Lasse S Vestergaard - lav@ssi.dk; Anja TR Jensen - atrj@cmp.dk; Trine Staalsoe - staalsoe@cmp.dk; Thor G Theander - Theander@cmp.dk; \\ Ib C Bygbjerg - i.bygbjerg@pubhealth.ku.dk; Michael Alifrangis - alifrangis@cmp.dk \\ * Corresponding author
}

Published: 16 November 2007

Malaria Journal 2007, 6:153 doi:10.1186/1475-2875-6-153
Received: 2I September 2007

Accepted: 16 November 2007

This article is available from: http://www.malariajournal.com/content/6/I/I53

(c) 2007 Enevold et al; licensee BioMed Central Ltd.

This is an Open Access article distributed under the terms of the Creative Commons Attribution License (http://creativecommons.org/licenses/by/2.0), which permits unrestricted use, distribution, and reproduction in any medium, provided the original work is properly cited.

\begin{abstract}
Background: In malaria endemic areas children may recover from malaria after chemotherapy in spite of harbouring genotypically drug-resistant Plasmodium falciparum. This phenomenon suggests that there is a synergy between drug treatment and acquired immunity. This hypothesis was examined in an area of moderately intense transmission of $P$. falciparum in Tanzania during a drug trail with sulphadoxine-pyrimethamine (SP) or amodiaquine (AQ).
\end{abstract}

Methods: One hundred children with uncomplicated malaria were treated with either SP or AQ and followed for 28 days. Mutations in parasite genes related to SP and AQ-resistance as well as human sickle cell trait and alpha-thalassaemia were determined using PCR and sequence-specific oligonucleotide probes and enzyme-linked immunosorbent assay (SSOP-ELISA), and IgG antibody responses to a panel of $P$. falciparum antigens were assessed and related to treatment outcome.

Results: Parasitological or clinical treatment failure (TF) was observed in $68 \%$ and $38 \%$ of children receiving $\mathrm{SP}$ or $\mathrm{AQ}$, respectively. In those with adequate clinical and parasitological response (ACPR) compared to children with TF, and for both treatment regimens, prevalence and levels of anti-Glutamate-rich Protein (GLURP)-specific IgG antibodies were significantly higher $(P<0.00 I)$, while prevalence of parasite haplotypes associated with $S P$ and $A Q$ resistance was lower $(P=0.02$ and $P=0.07$, respectively). Interestingly, anti-GLURP-IgG antibodies were more strongly associated with treatment outcome than parasite resistant haplotypes, while the $\lg G$ responses to none of the other II malaria antigens were not significantly associated with ACPR.

Conclusion: These findings suggest that GLURP-specific IgG antibodies in this setting contribute to clearance of drug-resistant infections and support the hypothesis that acquired immunity enhances the clinical efficacy of drug therapy. The results should be confirmed in larger scale with greater sample size and with variation in transmission intensity. 


\section{Background}

Plasmodium falciparum resistance to commonly available antimalarial drugs such as chloroquine (CQ), amodiaquine (AQ) sulphadoxine-pyrimethamine (SP) is now widespread in most malaria-endemic areas, including Tanzania $[1,2]$. It has been established that polymorphisms in the parasite dihydrofolate reductase $(d h f r)$, dihydropteroate synthetase (dhps) and chloroquine resistance transporter $(P f c r t)$ genes are associated with SP and CQ resistance, respectively in vitro $[3,4]$. Point mutations at positions N51I, C59R and S108N in the dhfr gene $[5,6]$ and at positions A437G and K540E in the dhps gene $[7,8]$ have shown to predict a reduced efficacy to SP in vivo. Likewise, the K76T mutation in the Pfcrt gene is a well described predictor of reduced parasite susceptibility to CQ [9], and to a lesser extent AQ [10]. The prevalence of these mutations has increased as a result of high drug pressure in most sub-Saharan countries in recent years (reviewed in $[2,11]$ ).

Patients infected with $P$. falciparum parasites carrying such drug-resistant mutations sometimes overcome infection after treatment [12]. The ability to recover has been associated with host age $[13,14]$ and transmission intensity $[15,16]$, reflecting an effect of acquired host immunity. From animal models it has also been established that immunity enhances the efficacy of malaria drug treatment [17]. Moreover, haemoglobinopathies, such as sickle cell trait, has been related to increased efficacy of SP treatment of uncomplicated falciparum malaria in Kenya [18]. Therefore, recovery from malaria may depend on the drug efficacy and parasite drug-resistance, as well as a complex interaction with host factors like acquired immunity and innate resistance e.g. haemoglobinopathies.

Various studies have investigated the relationship between potential immune mechanisms, such as antibody responses, and therapeutic efficacy. It has been demonstrated that increased amounts of anti-RESA and antiNANP antibodies in patients treated with CQ were associated with better clearance of resistant parasites $[19,20]$, whereas other studies could not establish evidence for elevated anti-MSP1 and anti-AMA1 antibody levels in patients recovering after treatment with CQ, SP or AQ [2123]. These observations are however difficult to compare, when factors like patient age, innate resistance, intensity of transmission and level of drug resistance vary substantially between these studies and may influence treatment outcome.

The objective of this study was to evaluate factors influencing outcome of antimalarial treatment to uncomplicated $P$. falciparum malaria, such as acquired immunity, haemoglobinopathies and genotypic markers of drug resistance. The study likewise wanted to investigate the applicability of drug efficacy trails in testing the importance of antibodies to different vaccine-candidates in patients receiving drugs with reduced efficacy, as suggested previously $[14,22]$. Patients were children below five years of age exposed to low-to-moderate levels of malaria transmission in Tanzania, treated with either SP or AQ for episodes of uncomplicated febrile malaria.

\section{Methods \\ Study population and samples}

The study was done as part of an annual clinical drug-efficacy trial under the East Africa Network for Monitoring Antimalarial Treatment (EANMAT) in collaboration with the National Malaria Control Programme in Tanzania. The trial was conducted during the rainy season between February and July 2005 in Chamwino village, Dodoma region, which is an area characterized by low-to-moderate malaria transmission of mainly $P$. falciparum. The study protocol was approved by the Ethical Committee of the National Institute for Medical Research and Ministry of Health, Tanzania.

The efficacy study were designed to enrol 100 patients aged 6-59 months presenting with uncomplicated malaria if they met the criteria as defined in the standard efficacy testing protocol by WHO [24]: i) monoinfection with $P$. falciparum at parasite densities above $2,000 / \mu \mathrm{l}$ and below $200,000 / \mu \mathrm{l}$, ii) axillary temperature $\geq 37.5^{\circ} \mathrm{C}$, iii) haemoglobin $>5 \mathrm{~g} / \mathrm{dl}, i v$ ) absence of severe malnutrition, $v$ ) absence of general danger signs, severe and complicated malaria, and $v i$ ) informed written consent from parents/guardians.

Patients were randomly allocated to receive either sulphadoxine-pyrimethamine (SP) $(25 \mathrm{mg} / \mathrm{kg}$ sulphadoxine and $1.25 \mathrm{mg} / \mathrm{kg}$ pyrimethamine) as a single dose (Fansidar $^{\circledast}$, Roche, Switzerland) or amodiaquine (AQ) $(10 \mathrm{mg} /$ $\mathrm{kg}$ ), once daily for three days (Pfizer, Senegal). Patients were followed up clinically and parasitologically on days $0,1,2,3,7,14$, and 28 and on any other day if the patient felt unwell.

Treatment outcome during 28 days of follow-up were classified as early treatment failure (ETF), late clinical failure (LCF), late parasitological failure (LPF) or adequate clinical and parasitological response (ACPR) according to the standard protocol of WHO [24]. For the purpose of this study, patients with ETF, LCF and LPF were grouped as treatment failures (TF). LPF were defined as patients with presence of parasitaemia on any day from day 7 to day 28 and axillary temperature $<37.5^{\circ} \mathrm{C}$ without previously having ETF or LCF. Patients failing treatment or with signs of severe malaria were given quinine and referred to the nearby district hospital. 
Parasitaemia was determined microscopically using thin smear of fingerprick blood and stained with 3\% Giemsa. Packed cell volume (PCV) was measured in haematocrit capillary tubes. Plasma samples were collected upon centrifugation, and stored at minus $20^{\circ} \mathrm{C}$ until further analysis.

\section{DNA and genetic analysis}

DNA was extracted from fingerprick blood collected on filter paper, as previously described [25]. Nested polymerase chain reaction (PCR) assays were applied to differentiate recrudescence from new $P$. falciparum infections by comparing PCR-generated $m s p 2$ genotype patterns in regions FC27 and IC-1 from samples collected on day 0, 7, 14 and 28 [26]. Analysis of the $m s p 2$ genotypes was done according to Cattamanchi et al [27]: An outcome was defined as recrudescence if a subsequent sample on any day from day 7 to day 28 post-treatment contained identical alleles or a subset of the alleles present in the day 0 sample and the patient were classified as having late parasitological treatment failure (LPF). An outcome was defined as re-infection if a subsequent sample on any day from day 7 to day 28 post-treatment contained only new alleles compared to the day 0 sample and the patient were classified as having an adequate clinical and parasitological response (ACPR) [24].

Plasmodium falciparum single nucleotide polymorphisms (SNPs) at dhfr (position 50/51, 59 and 108), dhps (position 436/437, 540, 581 and 613) and Pfcrt (position 72-76) were determined on day 0 by sequence-specific oligonucleotide probes (SSOP) and Enzyme-Linked ImmunoSorbent Assay (ELISA)-based technique of PCR amplified fragments as described in [27] Briefly, biotinconjugated nested PCR amplified DNA were fixed on streptavidin-coated ELISA plates and mixed with digoxigenin-labelled oligonucleotide probes with specificity for the SNP's of interest. The mixtures were washed with high stringency at set temperatures before incubated with peroxidase-conjugated anti-digoxigenin antibodies and visualized by o-phenylene-diamine (OPD). The SSOP's enables detection of mixed haplotypes with high specificity. The SNP's were constructed into haplotypes. Infections with mixed parasite haplotypes were only classified as 'mixed' when none of the polymorphisms in the same codon were dominant as determined by OD values of differences above 50\%. Sickle cell trait were detected by screening the human $\beta$-haemoglobin gene for the A18T mutation by SSOP-ELISA [28] and the African alpha ${ }^{3.7}$ deletion variant of alpha+-thalassaemia was determined as previously described [29].

\section{Recombinant P. falciparum antigens and synthetic peptides}

The $P$. falciparum antigens used in the present study were: the N-terminal non-repeat $\mathrm{R} 0$ region of the Glutamaterich Protein (GLURP-R0) (amino acids 27-500, FVO strain) [30]; the C-terminal GLURP-R2 repeat region (amino acids 705-1178, F32 strain) [31]; the synthetic peptide corresponding to the repeat region (NANPx6) of the circumsporozoite protein (CSP); the EBA-4 peptide of the Erythrocyte Binding Antigen-175 (EBA-175) [32]; the $\mathrm{N}$-terminal region of the Apical Membrane Protein 1 (AMA1) (amino acids 25 to 545, FVO strain) with mutated glycosylation sites [33]; the non-polymorphic Cterminal region of the Merozoite Surface Protein 3 (MSP3) (amino acids 212-380, FVO strain) [30]; the 19 kDa fragment of Merozoite Surface Protein 1 (MSP1) [34]; three PfEMP1 recombinant HIS-tagged proteins: The CIDR $1-\alpha$ and DBL4- $\gamma$-DBL5- $\delta$ domains of the PFD $1235 \mathrm{w}$ and the DBL2- $\beta$ domain of the PF13_0003 [35,36]; and crude extract of schizont material [37].

\section{Antibody levels estimated by ELISA}

The plasma samples collected at day 0 were tested for the presence of IgG to the eleven antigens mentioned above by ELISA as previously described [35]. Wells of Maxisorp microtiter plates (Nunc, Roskilde, Denmark) were coated with $100 \mu \mathrm{l}$ of the recombinant protein diluted in $0.1 \mathrm{M}$ glycine-HCl ( $\mathrm{pH} 2.75)$ (apart from CSP that was diluted in phosphate-buffered saline [PBS], $\mathrm{pH}$ 7.2) by overnight incubation at $4^{\circ} \mathrm{C}$. Plasma collected from 30 residents of a Tanzanian village where malaria is holoendemic were used as a reference positive plasma pool and plasma from 19 healthy Danish donors who have never been exposed to malaria were used as negative controls. To account for day-to-day variation between assays, antibody responses were calculated as arbitrary units (AU) with the following formula: [ $\left(\mathrm{OD}_{\text {sample plasma }}-\mathrm{OD}_{\text {background }}\right) /\left(\mathrm{OD}_{\text {positive plasma }}\right.$ $\left.\left.\mathrm{OD}_{\text {background }}\right)\right] \times 100$. The cut-of for a positive antibody response was defined as the mean level plus two standard deviations of the antibody reactivity among the negative controls.

\section{Measurement of VSA-specific IgG by flow cytometry}

Plasma levels of IgG with specificity for variant surface antigens (VSA) on two parasite isolate lines with different VSA-expression profiles, 3D7 unselected (VSA1) and 3D7 selected on transformed human bone marrow endothelial cells (THrMEB) (VSA2) [35] were tested by a flow cytometric assay previously described [38]. Plasma from six residents of a holoendemic Ghanaian village and six Danish donors served as positive and negative controls, respectively. For each plasma sample, the mean fluorescence index (MFI) was recorded and used as a measure of the VSA-specific antibody level. Antibody responses were calculated as arbitrary units (AU) with the following for- 
mula: $\left[\left(\mathrm{MFI}_{\text {sample plasma }}-\mathrm{MFI}_{\text {background }}\right) /\left(\mathrm{MFI}_{\text {positive plasma }}\right.\right.$ $\left.\left.\mathrm{MFI}_{\text {background }}\right)\right] \times 100$. The antibody positivity cut-of was determined as the mean antibody levels of the plasma from the mean of the six Danish donors plus two standard deviations.

\section{Statistics}

Statistical analyses of data were performed with Stata/SE version 8.2 (Stata Corp., Texas, US). $\chi^{2}$ test and Fisher's exact test were used to compare differences in proportions of antibody responders, presence of parasite resistant genotypes and genes assessing haemoglobinopathies in patients with adequate clinical and parasitological response (ACPR) and treatment failure (TF). Non-parametric Mann Whitney rank sum test was used to analyse differences in median antibody levels between treatment groups. Associations between quantitative variables as age, parasite densities and haemoglobin levels were assessed by linear regression. For multivariable analysis, logistic regression models were used to assess predictors of treatment failure. In these models, parasite density (log parasite density pre-treatment), age and age-squared were used as continuous exposure variables, while presence or absence of parasite resistant genotypes, human antibodies and genes causing haemoglobinopathies were included as categorical exposure variables. Possible effect modification between the exposure variables was considered by testing for interaction between presence of parasite mutant haplotypes and IgG antibodies. $\mathrm{P}<0.05$ was defined as significant.

\section{Results}

\section{Characteristics of the study groups and outcome of} treatment

The baseline characteristics of the 100 patients enrolled in the study are shown in Table 1. Age, sex, parasitaemia, temperature and haemoglobin levels on the day of enrolment were similar in the SP and AQ treatment groups.

Of the 50 patients receiving SP, none presented with early clinical failure (ECF), three patients presented with late clinical failures (LCF), 31 patients presented with late parasitological failures (LPF) and the remaining 16 patients had an adequate clinical and parasitological response (ACPR). Of the 50 patients receiving $A Q$, one presented with ECF, two patients presented with LCF, 16 patients presented with LPF and the remaining 31 patients had an ACPR. All samples were adjusted for new infections by PCR genotyping. Before PCR corrections, 32 patients receiving SP and 22 patients receiving AQ presented with LPF, respectively. All patients with either ECR, LCF or LPF were grouped together as treatment failures (TF). Thus, the treatment failure rates were $68 \%$ in the SP group and $38 \%$ in the AQ group. There were no significant differences with respect to age, sex, temperature, and haemoglobin level between patients with ACPR or TF in any of the treatment groups. However, in patients receiving SP, the parasite density on day 0 was higher in the TF than ACPR group $(P=0.03)$. Levels of haemoglobin, parasite density and failure rates showed no relation to age (data not shown).

\section{Associations between parasite mutant haplotypes and treatment outcome}

The haplotypes based on the single nucleotide polymorphisms at codon 50/51, 59 and 108 of the P. falciparum dhfr gene, and codon 436/437, 540, 581 and 613 of the $P$. falciparum dhps gene, were determined on day 0 for parasite infections in patients receiving SP (Table 2).

A higher prevalence of the triple mutant haplotype, CIRN in $d h f r$, was observed in patients experiencing TF $(82 \%)$ compared to patients with ACPR $(50 \%),\left(\chi^{2}=5.6, \mathrm{P}=\right.$

Table I: Baseline characteristics of patients.

\begin{tabular}{|c|c|c|c|c|}
\hline \multirow[b]{2}{*}{ Treatment outcome } & \multicolumn{2}{|c|}{$S P(n=50)$} & \multicolumn{2}{|c|}{$A Q(n=50)$} \\
\hline & TF & ACPR & TF & ACPR \\
\hline PCR un-corrected, N (\%) & $35(70)$ & $15(30)$ & $22(44)$ & $28(56)$ \\
\hline PCR corrected, $\mathrm{N}(\%)$ & $34(68)$ & $16(32)$ & $19(38)$ & $31(62)$ \\
\hline Median age (months, $\mathrm{Cl}$ ) & $31.5(25.3-36)$ & $27.5(19-41.4)$ & $24(19.4-43.9)$ & $32(23.5-38.5)$ \\
\hline Females (\%) & 31.6 & 38.7 & $4 I .2$ & 68.7 \\
\hline $\begin{array}{l}\text { Geometric mean parasite density, day0 } \\
\text { (/ul. Cl) }\end{array}$ & $39.810(28.184-56.234)^{*}$ & $17.865(8.5 \mid I-37.153)$ & $28.840(14.256-57.544)$ & $32.359(22.387-43.65 \mathrm{I})$ \\
\hline Mean haemoglobin day0 (PCV, SD) & $29.4(4.8)$ & $29.6(5.8)$ & $28.6(6.5)$ & $30.7(5.1)$ \\
\hline Mean temperature day $0\left({ }^{\circ} \mathrm{C} . \mathrm{SD}\right)$ & $38.8(0.9)$ & $38.5(0.6)$ & $38.5(0.9)$ & $39.1(0.8)$ \\
\hline Alpha $^{+}$-thalassaemia, $\%$ & 33.4 & 27.6 & 42.9 & 35.7 \\
\hline $\mathrm{HbAS}, \%$ & 15.2 & 12.5 & 10.4 & 9.7 \\
\hline
\end{tabular}

TF: Parasitological or clinical treatment failure, ACPR: Adequate clinical and parasitological response. SP: sulphadoxine-pyrimethamine, AQ: amodiaquine. * Significantly different from value for parasite density in ACPR group as calculated by Student's $t$ test. 
Table 2: Frequency of $P$. falciparum polymorphisms in dhfr, dhps and crt genes in relation to treatment outcome

\begin{tabular}{|c|c|c|c|}
\hline SP & Haplotypes & TF & ACPR \\
\hline $\mathrm{N}$ & & 34 & 16 \\
\hline \multicolumn{4}{|l|}{ dhfr } \\
\hline wildtype & CNCS & 0 & 2 \\
\hline SI08N, single & $\mathrm{CNCN}$ & 1 & 1 \\
\hline C59R + SI08N, double & CNRN & I & 1 \\
\hline N5II + SI08N, double & $\mathrm{CICN}$ & 3 & 3 \\
\hline $\mathrm{N} 5 \mathrm{II}+\mathrm{C} 59 \mathrm{R}+\mathrm{SI} 08 \mathrm{~N}$, triple & CIRN & 28 & 8 \\
\hline Mixed* & & 1 & 1 \\
\hline \multicolumn{4}{|l|}{ dhps } \\
\hline wildtype & SAKAA/AAKAA & 18 & 11 \\
\hline A437G, single & SGKAA & 5 & 0 \\
\hline A437G + K540E, double & SGEAA & II & 4 \\
\hline single-double combined & & 16 & 4 \\
\hline Mixed* & & 0 & 1 \\
\hline \multicolumn{4}{|l|}{ dhfr-dhps } \\
\hline triple-double & & 10 & 3 \\
\hline triple-single & & 4 & 0 \\
\hline double-double & & 1 & 1 \\
\hline AQ & & TF & ACPR \\
\hline $\mathrm{N}$ & & 19 & 31 \\
\hline $76 \mathrm{~K}$ & CVNMK & 3 & 12 \\
\hline $76 \mathrm{~T}$ & CVIET & 16 & 18 \\
\hline Mixed* & & 0 & 1 \\
\hline
\end{tabular}

TF: Parasitological or clinical treatment failure, ACPR: Adequate clinical and parasitological response. SP: sulphadoxine-pyrimethamine, AQ: amodiaquine. Haplotypes defined as amino acid sequences based on the following substitutions in dhfr: SI08N (single), C59R + SI08N or N5II + C59R (double) and N5II + C59R + SI08N (triple), and in dhps: A437G (single) and A437G + K540E (double) were assessed. For the crt gene, the K76T amino acid substitution was assessed.

*Classified as mixed when none of the haplotypes were dominant.

0.02). There was no significant association between $d h p s$ mutant haplotypes (single SGKAA and double SGEAA combined) and treatment outcome $\left(\chi^{2}=1.8, \mathrm{P}=0.18\right)$, nor between the quintuple $d h f r-d h p s$ mutant haplotype (CIRN-SGEAA) and TF $\left(\chi^{2}=0.6, P=0.46\right)$. However, due to the low number of samples, we could not exclude a possible association between the quintuple mutant haplotype and treatment outcome.

The majority of parasites in patients receiving $A Q$ expressed the Pfcrt mutant haplotype CVIET (68\%) and the remaining samples were the CVMNK wildtype haplotype. There was a trend towards higher prevalence of the CVIET haplotype in patients failing treatment (84\%) compared to patients with ACPR (58\%), although not statistically significant $\left(\chi^{2}=3.2, \mathrm{P}=0.08\right)$.
Thus, a higher prevalence of parasites expressing triple mutant $d h f r$ haplotype in patients failing SP treatment and a tendency for a higher prevalence of the mutant CVIET haplotype in patients failing AQ treatment were observed, indicating some association between presence of parasite resistant genotypes and treatment outcome.

\section{Associations between anti-malaria antibodies and treatment outcome}

Prevalence and levels of IgG antibodies with specificity for the eleven malaria antigens and the surface of the two parasite isolates were measured on day 0 in patients treated with SP or AQ (Figure 1 and Table 3). In univariate analysis, patients with ACPR had a higher prevalence of antiGLURP-R0 IgG $\left(\chi^{2}=20.9, P<0.001\right)$ and anti-GLURP-R2 $\operatorname{IgG}\left(\chi^{2}=12.7, \mathrm{P}<0.001\right)$ as well as higher levels of antiGLURP-R0 and R2 IgG $(\mathrm{P}<0.001)$ compared to patients with TF. Such statistically significant associations were not observed for any of the other antigen-specific IgG responses. Furthermore, the associations between ACPR and prevalence and levels of GLURP-R0 and R2 antibodies were significant when the SP and AQ groups were analysed separately ([SP]: R0: $\chi^{2}=11.8, \mathrm{P}=0.001 ; \mathrm{R} 2: \chi^{2}=9.4$, $\mathrm{P}=0.002$. [AQ]: R0: $\chi^{2}=11.3, \mathrm{P}=0.001 ; \mathrm{R} 2: \chi^{2}=3.3, \mathrm{P}=$ $0.07)$. The prevalence of the combined IgG responses to GLURP-R0 and R2 was also correlated to ACPR in both the SP $\left(\chi^{2}=10.1, P=0.002\right)$ and the AQ $\left(\chi^{2}=11.3, P=\right.$ $0.001)$ group. By contrast, no association between antibody responses to any other antigen, analysed individually or in combination, and treatment outcome in neither the SP nor the AQ group was demonstrated. Approximately $25 \%$ of patients with IgG against GLURP R0 did not have IgG against R2 and vice versa, indicating that responding to R0 not necessarily means responding to R2. Thus, among the thirteen IgG specificities, only antiGLURP-R0 and R2 IgG proved to be associated with ACPR.

\section{Impact of haemoglobinopathies on anti-malaria antibodies, parasitaemia and treatment outcome}

In the study population, the overall frequency of homozygote and heterozygote alpha+-thalassaemia was $38 \%$ and the frequency of sickle cell trait (HbAS) 12\%. No sickle cell anaemic patients (HbSS) were found. The prevalence of these traits was similar in the two treatment outcome groups for both SP and AQ (see Table 1). Prevalence and levels of antibodies did not differ between patients with and without sickle cell trait or with and without alpha+thalassaemia for any of the malaria antigens. A lower parasite density was observed at enrolment in patients with alpha $^{+}$-thalassaemia receiving AQ $(P=0.007)$, but not for SP $(P=0.41)$. 


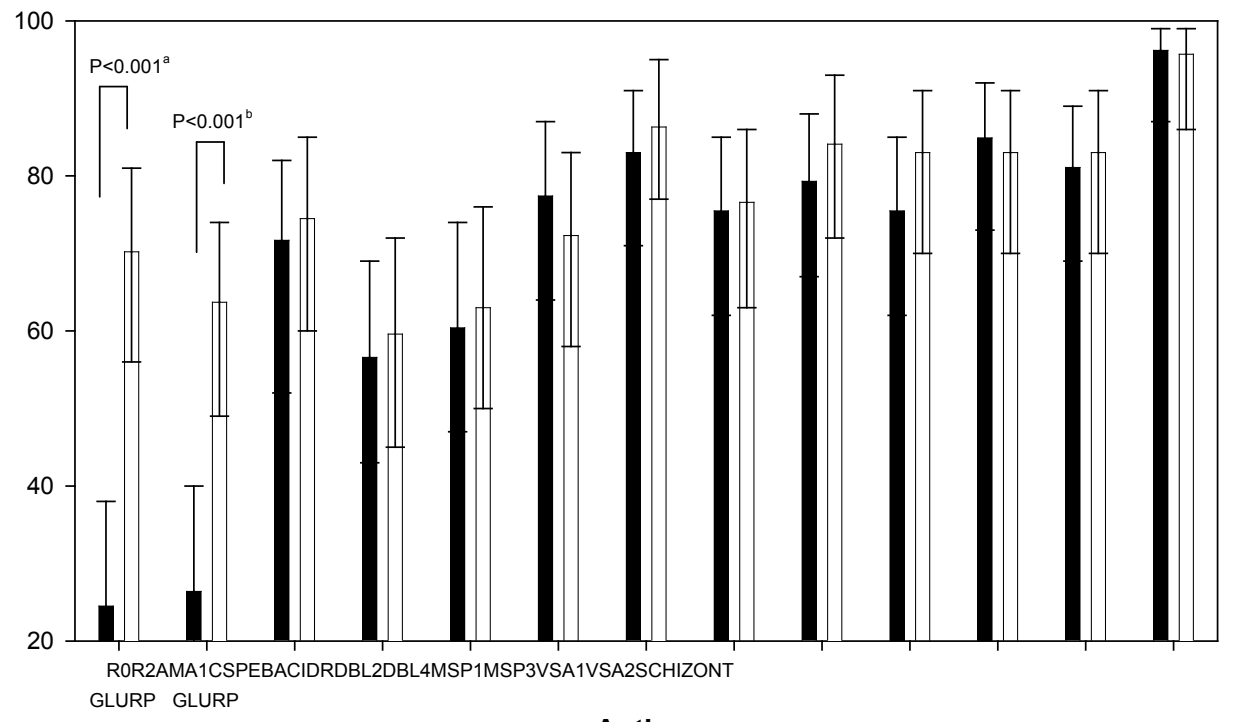

Antigen

\section{Figure I}

Prevalence of IgG antibody in patients at day 0 to different malaria antigens in relation to treatment outcome. Black: Parasitological or clinical treatment failure (TF), White: Adequate clinical and parasitological response (ACPR). Patients receiving sulphadoxine-pyrimethamine (SP) or amodiaquine (AQ) are grouped together. Error bars illustrate $95 \%$ confidence intervals. $\chi^{2}$ test were done to compare proportions of $\operatorname{lgG}$ antibody responders between treatment outcome groups. Only significant $p$-values are presented. aGLURP-R0; $\chi^{2}=20.9$, b GLURP-R2; $\chi^{2}=12.7$. GLURP-R0: Glutamate-rich Protein region Nterminal, GLURP-R2: Glutamate-rich Protein region C-terminal, AMAI: Apical Membrane Antigen I, CSP: Circumsporozoite Protein, EBA: Erythrocyte Binding Antigen- 175, CIDR: CIDR domain of PfEMPI (PFDI235w), DBL2: DBL2 domain of PfEMPI (PFI3_0003), DBL4: DBL4 domain of PfEMPI (PFDI235w), MSPI: Merozoite Surface Protein I, MSP3: Merozoite Surface Protein 3, VSAI: Variant Surface Antigen on unselected 3D7 parasite strain, VSA2: Variant Surface Antigen on bone marrow selected 3D7 parasite strain. SCHIZONT: Crude extract of schizont material.

\section{Potential predictors of treatment outcome}

Patients were divided into four groups based on infections with or without mutant haplotypes (the $d h f r$ triple mutant haplotype in the SP group, and the CVIET haplotype in the AQ group), and presence or absence of IgG antibodies to each antigen. In Table 4, only data for GLURP-R0 and R2 are shown.

As expected, patients with anti-GLURP IgG infected with a sensitive haplotype (group 1) were more likely to have ACPR compared to patients with no anti-GLURP IgG infected with a resistant parasite haplotype (group 4). Interestingly, there was a marked positive effect of having anti-GLURP IgG on treatment outcome in patients with resistant haplotypes (comparing group 3 vs. 4 for GLURPR0; $[S P]: R 0, P=0.004$ and $[A Q]: R 0, P=0.02)$, indicating that possessions of anti-GLURP IgG were associated with reduced risk of $\mathrm{TF}$ when infected with resistant parasites. Similar significant associations were seen for GLURP-R2 but not for any of the other antigens.

In multivariate regression models, the effect of IgG antibodies, parasite haplotype and parasite density on treat- ment outcome was analysed (Table 5). Regardless of parasite density and age, anti-GLURP IgG were the strongest predictor of treatment outcome in the SP and AQ groups separately and combined. Presence of resistant parasite haplotypes and high parasitaemia were also associated with treatment failure in patients receiving SP, but not as strongly as the presence of GLURP-R0 and R2 IgG and not in the AQ group.

\section{Discussion}

This study evaluated the contribution of some antimalaria antibodies and parasite resistant haplotypes on the outcome of SP and AQ treatment of patients under five years of age with uncomplicated malaria. The rate of SP treatment failure observed (68\%) is unexpectedly high for this area of endemicity, but similar SP failure rates $(43 \%-74 \%)$ have been shown in other parts of Tanzania the last 10 years [39-41]. Not surprisingly patients failing SP and AQ treatment were generally infected with parasites expressing drug resistance-related mutations. However, increased risk of SP treatment failure was associated with the triple $d h f r$ (CIRN) irrespective of the dhps haplotype status as demonstrated in other studies [5,42]. In the 
Table 3: Level of IgG antibodies in patients to different malaria antigens in relation to treatment outcome.

\begin{tabular}{|c|c|c|c|}
\hline \multirow[b]{2}{*}{ Antigen } & \multicolumn{2}{|c|}{ Median level ( $25^{\text {th }}$ and $75^{\text {th }}$ percentiles)* } & \multirow[b]{2}{*}{ P-value } \\
\hline & $\mathrm{TF}, \mathrm{n}=53$ & $\mathrm{ACPR}, \mathrm{n}=47$ & \\
\hline GLURP-RO & $0.0(0.0-1.3)$ & $8.4(0.0-47.7)$ & $<0.001$ \\
\hline GLURP-R2 & $0.0(0.0-5.0)$ & $7.7(0.0-49.8)$ & $<0.001$ \\
\hline AMA-I & $24.3(0.0-51.1)$ & $24.5(0.0-87.3)$ & 0.25 \\
\hline CSP & $5.8(0.0-17.1)$ & $10.2(0.0-24.4)$ & 0.42 \\
\hline CIDRI $\alpha$ & $15.4(3.3-61.7)$ & $9.1(0.0-30.2)$ & 0.24 \\
\hline $\mathrm{DBL} 2 \beta$ & $22.5(6.9-45.6)$ & $16.9(3.4-58.3)$ & 0.92 \\
\hline DBL4 $4-D B L 5 \delta$ & $10.4(1.6-38.6)$ & $12.4(3.0-44.0)$ & 0.74 \\
\hline MSP-I & $80.0(6.7-144.4)$ & I $22.7(29.3-162.0)$ & 0.11 \\
\hline MSP-3 & $5.3(1.1-12.6)$ & $8.0(2.5-18.9)$ & 0.30 \\
\hline VSAI & $15.5(5.2-33.0)$ & $19.8(6.1-4 \mid .0)$ & 0.58 \\
\hline VSA2 & $16.3(4.1-30.9)$ & $21.1(7.6-38.7)$ & 0.21 \\
\hline EBA-175 & $4.3(0.0-14.1)$ & $5.9(0.0-21.0)$ & 0.54 \\
\hline SCHIZONT & $30.0(|2.9-5| .0)$ & $35.2(11.1-80.2)$ & 0.17 \\
\hline
\end{tabular}

TF: Parasitological or clinical treatment failure, ACPR: Adequate clinical and parasitological response. Patients receiving sulphadoxinepyrimethamine (SP) or amodiaquine (AQ) are grouped together. *Median values of lgG level day 0 in arbitrary units $\left(25^{\text {th }}\right.$ and $75^{\text {th }}$ percentiles). $P$-values determined by Mann-Whitney rank-sum test. Similar tendencies were observed when restricting the analysis to those with a measurable antibody level only, and when analysing each drug arm separately. For definitions of antigens, see Figure $I$ and in the method section.

Table 4: Associations between presence of IgG antibodies to GLURP-R0 + R2 and parasite haplotypes in relation to treatment outcome.

\begin{tabular}{|c|c|c|c|c|c|}
\hline \multirow[b]{2}{*}{$\begin{array}{l}\text { Treatment } \\
\text { and group SP }\end{array}$} & \multirow[b]{2}{*}{$\begin{array}{l}\text { Presence of antibody/ } \\
\text { resistant haplotype* }\end{array}$} & \multicolumn{2}{|c|}{ GLURP-RO } & \multicolumn{2}{|c|}{ GLURP-R2 } \\
\hline & & $\mathrm{TF}$ & ACPR & $\mathrm{TF}$ & ACPR \\
\hline 1 & $+/-$ & 2 & 4 & 1 & 6 \\
\hline 2 & $-/-$ & 4 & 3 & 5 & 2 \\
\hline 3 & $+/+$ & 6 & 7 & 9 & 7 \\
\hline 4 & $-/+$ & 22 & 2 & 19 & 1 \\
\hline \multicolumn{6}{|l|}{$\mathrm{AQ}$} \\
\hline 1 & $+/-$ & 1 & 7 & 0 & 8 \\
\hline 2 & $-/-$ & 2 & 5 & 3 & 4 \\
\hline 3 & $+/+$ & 4 & 12 & 3 & 11 \\
\hline 4 & $-/+$ & 12 & 6 & 13 & 7 \\
\hline
\end{tabular}

TF: Parasitological or clinical treatment failure, ACPR: Adequate clinical and parasitological response. SP: sulphadoxine-pyrimethamine, AQ: amodiaquine. *Number of patients with presence $(+)$ or absence (-) of IgG antibodies to GLURP-R0 or R2 combined with resistant (+) or sensitive (-) genotypes associated with treatment outcome. Haplotypes were defined as resistant if parasites expressed the $\mathrm{dhfr}$ triple haplotype (CIRN) for the SP group or the CVIET haplotype for the $\mathrm{AQ}$ group.
AQ treatment group, only a trend towards an increased risk of treatment failure was found in patients infected with the Pfcrt-CVIET mutant haplotype. Although the latter could be explained by involvement of other putative genes related to AQ resistance, such as Pfmdr [43], and that the assumptions are based on a relatively small sample size, these findings indicate that the association between parasite resistant genotypes and SP or AQ treatment outcome is not absolute.

Interestingly, the prevalence and level of anti-GLURP antibodies was associated with a substantial decreased risk of treatment failure irrespective of drug regimen. This association could not be demonstrated for any of the other antigens studied, neither individually nor combined. Similarly, although there was an association between parasite density and patients failing SP treatment, the treatment outcome was independent of age, alpha ${ }^{+}$ thalassaemia (homozygote or heterozygote) and sickle cell trait, and for AQ treatment outcome, also parasite density. Furthermore, in regression analysis, the presence of GLURP R0 or R2 specific IgG antibodies influenced the risk for failing SP and AQ treatment more strongly than presence of parasite resistant haplotypes. This is in line with a recent study from Uganda, where transmission intensity, rather than the parasite genotype, influenced treatment outcome [16]. This indicates that the degree of immunity in the population in combination with the number of resistant-related parasite mutations predicts ACPR, suggesting a synergistic effect between the antimalarial and host immunity, as also demonstrated in mice [17].

It has recently been proposed that therapeutic responses to resistant malaria infections can provide a mechanism for measuring effective clinical immunity [14], i.e. drug trials can be useful in identifying antigens and vaccine candidates affording protection [22]. Investigations of the impact of immunity on treatment outcome have however resulted in conflicting findings [19-23]. In line with the observations from the present study, level of anti-IgG to $\mathrm{MSP}_{19}$ was not associated with CQ [22] SP [23] or AQ $[21,23]$ treatment outcome, and prevalence of anti-AMA1 [22] and anti-MSP3 [19] IgG was not associated with reduced risk for CQ treatment failure. However, in contrast to the findings from the present study, the prevalence of IgG to $M S P 1_{1}$ was higher in patients clinically recovering after CQ treatment [22], and IgG reactivity to multiple, but not single, K1 and MAD20 alleles of MSP1 were related to efficacy of AQ treatment [21]. In addition, prevalence of anti-NANP and anti-RESA IgG has been demonstrated to be higher in patients recovering successfully from CQ [19] and artesunate [20] treatment. The inability to demonstrate an association between prevalence and levels of antibodies against antigens other than GLURP in 
Table 5: Predictors for treatment failure.

\begin{tabular}{|c|c|c|c|c|c|}
\hline Treatment group & Risk variables & Unadjusted OR $(95 \% \mathrm{Cl})$ & $\mathrm{p}$-value & Adjusted OR $(95 \% \mathrm{Cl})^{*}$ & $\mathrm{p}$-value \\
\hline \multirow[t]{3}{*}{$S P(n=50)$} & Parasite density & $3.74(1.12-12.54)$ & 0.03 & $3.16(0.73-13.71)$ & 0.13 \\
\hline & CIRN & $4.67(1.25-17.44)$ & 0.02 & 7.21 (1.42-36.73) & 0.02 \\
\hline & GLURP-R0 & $0.09(0.02-0.41)$ & 0.002 & $0.08(0.14-0.47)$ & 0.005 \\
\hline \multirow[t]{3}{*}{$\mathrm{AQ}(\mathrm{n}=50)$} & Parasite density & $0.82(0.27-2.46)$ & 0.72 & $0.77(0.13-4.42)$ & 0.76 \\
\hline & CVIET & $3.55(0.85-14.91)$ & 0.08 & $2.56(0.46-14.27)$ & 0.28 \\
\hline & GLURP-RO & $0.10(0.02-0.43)$ & 0.002 & $0.07(0.01-0.48)$ & 0.003 \\
\hline \multirow[t]{3}{*}{$S P+A Q(n=100)$} & Parasite density & $1.6 \mathrm{I}(0.74-3.47)$ & 0.23 & $1.13(0.43-2.99)$ & 0.80 \\
\hline & CIRN/CVIET & 3.76 (1.49-9.47) & 0.005 & $3.44(1.12-10.54)$ & 0.03 \\
\hline & GLURP-RO & $0.13(0.06-0.33)$ & $<0.001$ & $0.11(0.04-0.31)$ & $<0.001$ \\
\hline
\end{tabular}

Multivariate regression models predicting failure of antimalarial treatment. SP: sulphadoxine-pyrimethamine, $\mathrm{AQ}$ : amodiaquine.OR: Odds Ratio, Cl: 95\% Confidence interval. CIRN: Presence of the triple dhfr CIRN haplotype. CVIET: Presence of the crt CVIET haplotype. CIRN/CVIET: Presence of parasites with CIRN in patients treated with SP and presence of parasites with CVIET in patients treated with AQ. GLURP-R0: Presence of IgG antibodies to GLURP-R0. SP+AQ: Total patients in the two treatment groups. *Adjusted for age, parasite density, presence of mutant haplotypes and GLURP-RO antibodies. No interaction between presence of mutant haplotypes and GLURP-RO IgG antibodies was detected.

the present study may be explained by the relatively small sample size ( 50 patients in each group); alternatively by allelic diversity as shown in the study from Gabon (20), or by variable IgG subclass composition of the patient sera. For instance, it has previously been described that only IgG3 against MSP3 is associated with protection against clinical malaria [44], although this was not observed in another study where total IgG was a strong predictor of protection [45]. Finally, one could speculate if prevalence or level of IgG antibodies to these other specific antigens are not primary responsible for, or associated with, host immune protection against uncomplicated malaria but rather severe malaria - which none of the patients had. However, in patients with established clinical malaria, sporozoite-blocking anti-CSP antibodies were, however, not expected to influence treatment outcome.

Interestingly, at day 0 , all patients had IgG antibodies to at least one of the antigens tested, but no patient had IgG antibodies to all the antigens. This may reflect substantial variation in response to infections. Previous immuno-epidemiological studies have demonstrated that GLURP-specific IgG antibodies are associated with protection against high parasitaemia [46], clinical disease [47] and inhibition of parasite growth in vitro [48], but this is not direct evidence of IgG antibodies against GLURP increase the ability to clear uncomplicated malaria infections, nor of a causal relationship between treatment success and $\operatorname{IgG}$ antibodies against GLURP. However, it was shown that patients clinically recovering after treatment are more likely to carry anti-GLURP IgG antibodies and the presence of such antibodies were associated with reduced risk of failing drug-resistant parasite infections.

\section{Conclusion}

This study suggests that presence or level of GLURP-specific IgG antibodies in some settings are better predictors of SP and AQ treatment outcome than the parasite density at enrolment and drug-resistant related parasite mutations. This confirms the hypothesis that acquired immunity enhances the efficacy of antimalarial treatment, and supports the use of drug trials to identify markers of immunological importance. In addition, the findings suggest that drugs with reduced efficacy, such as SP, may still be effective for treatment of uncomplicated malaria in individuals with some acquired immunity, such as older children and adults living in areas of intense malaria transmission.

\section{Authors' contributions}

$\mathrm{AE}, \mathrm{LV}, \mathrm{MT}$ and MA conceived and designed the study. $\mathrm{WN}$ was responsible for the clinical trial and the supervision of patient enrolment and sample collection. AE performed the experiments and analysis with MA, MT, IB and LV. TS, AJ, IB and TT participated in manuscript preparation and design of the study. All authors read and approved the final manuscript.

\section{Acknowledgements}

The study was funded by the ENRECA, grant number 104.Dan.8.L.3 12 and the National Malaria Control Programme, Ministry of Health, Tanzania. AE is supported by DANIDA, grant number 91203.

The study was conducted under the auspices of the Joint Malaria Programme, a collaborative research initiative between Centre for Medical Parasitology at the University of Copenhagen, Kilimanjaro Christian Medical College, London School of Hygiene and Tropical Medicine and the Tanzania National Institute for Medical Research. We highly appreciate Dr Mutabingwa's clinical contributions and the help of technicians, nurses and clinical officers at the Chamwino Health Center in conducting the clinical trial and collecting the samples. We thank Kai Olsen for excellent technical 
laboratory assistance as well as Dr. Ed Remarque, Institute Pasteur, Paris, for providing the AMA-I antigen and Dr. Giampietro Corradin, University of Lausanne, Switzerland, for providing the CSP synthetic peptide.

\section{References}

I. Rapuoda B, Ali A, Bakyaita N, Mutabingwa TK, Mwita A, Rwagacondo C, Mugisha V, Staedke S, Snow RW, Watkins WM: The efficacy of antimalarial monotherapies, sulphadoxine-pyrimethamine and amodiaquine in East Africa: implications for sub-regional policy. Trop Med Int Health 2003, 8:860-867.

2. Wongsrichanalai C, Pickard AL, Wernsdorfer WH, Meshnick SR: Epidemiology of drug-resistant malaria. Lancet Infect Dis 2002, 2:209-218.

3. Fidock DA, Nomura T, Talley AK, Cooper RA, Dzekunov SM, Ferdig MT, Ursos LMB, Sidhu ABS, Naude B, Deitsch KW: Mutations in the $P$-falciparum digestive vacuole transmembrane protein PfCRT and evidence for their role in chloroquine resistance. Mol Cell 2000, 6:86I-87I.

4. Plowe CV, Cortese JF, Djimde A, Nwanyanwu OC, Watkins WM, Winstanley PA, EstradaFranco JG, Mollinedo RE, Avila JC, Cespedes $\mathrm{JL}$ : Mutations in Plasmodium falciparum dihydrofolate reductase and dihydropteroate synthase and epidemiologic patterns of pyrimethamine-sulfadoxine use and resistance. J Infect Dis 1997, I 76:I590-I596.

5. Basco LK, Tahar R, Keundjian A, Ringwald P: Sequence variations in the genes encoding dihydropteroate synthase and dihydrofolate reductase and clinical response to sulfadosinepyrimethamine in patients with acute uncomplicated falciparum malaria. J Infect Dis 2000, I 82:624-628.

6. Nzila AM, Nduati E, Mberu EK, Sibley CH, Monks SA, Winstanley PA, Watkins WM: Molecular evidence of greater selective pressure for drug resistance exerted by the long-acting antifolate pyrimethaminel/sulfadoxine compared with the shorter-acting chlorproguanil/dapsone on Kenyan Plasmodium falciparum. J Infect Dis 2000, I 8 I:2023-2028.

7. Kublin JG, Dzinjalamala FK, Kamwendo DD, Malkin EM, Cortese JF, Martino LM, Mukadam RAG, Rogerson SJ, Lescano AG, Molyneux ME: Molecular markers for failure of sulfadoxine-pyrimethamine and chlorproguanil-dapsone treatment of Plasmodium falciparum malaria. J Infect Dis 2002, 185:380-388.

8. Kyabayinze D, Cattamanchi A, Kamya MR, Rosenthal PJ, Dorsey G: Validation of a simplified method for using molecular markers to predict sulfadoxine-pyrimethamine treatment failure in African children with falciparum malaria. Am J Trop Med Hyg 2003, 69:247-252.

9. Wellems TE, Plowe CV: Chloroquine-resistant malaria. J Infect Dis 2001, I 84:770-776.

10. Ochong EO, Van den Broek IVF, Keus K, Nzila A: Short report: Association between chloroquine and amodiaquine resistance and allelic variation in the Plasmodium falciparum multiple drug resistance I gene and the chloroquine resistance transporter gene in isolates from the upper Nile in southern Sudan. Am J Trop Med Hyg 2003, 69:184-187.

II. Wernsdorfer WH, Noedl H: Molecular markers for drug resistance in malaria: use in treatment, diagnosis and epidemiology. Curr Opin Infect Dis 2003, 1 6:553-558.

12. Dorsey G, Kamya MR, Singh A, Rosenthal PJ: Polymorphisms in the Plasmodium falciparum pfcrt and pfmdr-I genes and clinical response to chloroquine in Kampala, Uganda. J Infect Dis 2001, I 83:1417-1420.

13. Omar SA, Adagu IS, Warhurst DC: Can pretreatment screening for dhps and dhfr point mutations in Plasmodium falciparum infections be used to predict sulfadoxine-pyrimethamine treatment failure? Trans R Soc Trop Med Hyg 200I, 95:3I5-3I9.

14. Djimde AA, Doumbo OK, Traore $O$, Guindo $A B$, Kayentao $K$, Diourte Y, Niare-Doumbo S, Coulibaly D, Kone AK, Cissoko Y, et al.: Clearance of drug-resistant parasites as a model for protective immunity in Plasmodium falciparum malaria. Am J Trop Med Hyg 2003, 69:558-563.

15. Khalil IF, Alifrangis M, Tarimo DS, Staalso T, Satti GMH, Theander TG, Ronn AM, Bygbjerg IC: The roles of the pfort $76 \mathrm{~T}$ and pfmdrl $86 Y$ mutations, immunity and the initial level of parasitaemia, in predicting the outcome of chloroquine treatment in two areas with different transmission intensities. Ann Trop Med Parasitol 2005, 99:44I-448.
16. Francis D, Nsobya SL, Talisuna A, Yeka A, Kamya MR, Machekano R, Dokomajilar C, Rosenthal PJ, Dorsey G: Geographic differences in antimalarial drug efficacy in Uganda are explained by differences in endemicity and not by known molecular markers of drug resistance. J Infect Dis 2006, 193:978-986.

17. Targett GAT: Malaria - Drug-Use and the Immune-Response. Parasitology 1992, I05:S6I-S70.

18. Terlouw DJ, Aidoo MA, Udhayakumar V, Kolczak MS, Oloo AJ, Kager PA, Lal AA, Nahlen BL, ter Kuile FO: Increased efficacy of sulfadoxine-pyrimethamine in the treatment of uncomplicated falciparum malaria among children with sickle cell trait in western Kenya. J Infect Dis 2002, I86: |66|-1668.

19. Robert V, Roeffen W, Brasseur P, Aribot G, Verhave JP, Roussilhon $C$ : Anti-NANP antibody and treatment efficacy in patients with acute uncomplicated falciparum malaria attacks. Parasite Immunol 2000, 22:589-593.

20. Mayxay M, Chotivanich K, Pukrittayakamee S, Newton P, Looareesuwan S, White NJ: Contribution of humoral immunity to the therapeutic response in falciparum malaria. Am J Trop Med Hyg 2001, 65:918-923.

21. Mawili-Mboumba DP, Borrmann S, Cavanagh DR, McBride JS, Matsiegui PB, Missinou MA, Kremsner PG, Ntoumi F: Antibody responses to Plasmodium falciparum merozoite surface protein-I and efficacy of amodiaquine in gabonese children with P-falciparum malaria. J Infect Dis 2003, I87: I I37-I I4I.

22. Pinder M, Sutherland C], Sisay-Joof F, Ismaili J, Mccall MBB, Ord R, Hallett R, Holder AA, Milligan P: Immunoglobulin $G$ antibodies to merozoite surface antigens are associated with recovery from chloroquine-resistant Plasmodium falciparum in gambian children. Infect Immun 2006, 74:2887-2893.

23. Aubouy A, Migot-Nabias F, Deleron P: Correlations between treatment outcome and both anti-MSPI antibody response and erythrocyte-related genetic factors in Plasmodium falciparum malaria. Infect Genet Evol 2007, 7: |47-I54.

24. World Health Organization: Assessment and monitoring of antimalarial drug efficacy for the treatment of uncomplicated falciparum malaria. WHO: Geneva 2003.

25. Pearce RJ, Drakeley C, Chandramohan D, Mosha F, Roper C: Molecular determination of point mutation haplotypes in the dihydrofolate reductase and dihydropteroate synthase of Plasmodium falciparum in three districts of northern Tanzania. Antimicrob Agents Chemother 2003, 47:1347-1354.

26. Snounou G, Zhu XP, Siripoon N, Jarra W, Thaithong S, Brown KN, Viriyakosol S: Biased distribution of $m s p l$ and $m s p 2$ allelic variants in Plasmodium falciparum populations in Thailand. Trans $R$ Soc Trop Med Hyg 1999, 93:369-374.

27. Alifrangis M, Enosse S, Pearce R, Drakeley C, Roper C, Khalil IF, Nkya WMMM, Ronn AM, Theander TG, Bygbjerg IC: A simple, highthroughput method to detect Plasmodium falciparum single nucleotide polymorphisms in the dihydrofolate reductase, dihydropteroate synthase, and $P$. falciparum chloroquine resistance transporter genes using polymerase chain reaction- and enzyme-linked immunosorbent assay-based technology. Am J Trop Med Hyg 2005, 72:155-162.

28. Enevold A, Vestergaard LS, Lusingu J, Drakeley CJ, Lemnge MM, Theander TG, Bygbjerg IC, Alifrangis M: Rapid screening for glucose6-phosphate dehydrogenase deficiency and haemoglobin polymorphisms in Africa by a simple high-throughput SSOP. ELISA method. Malar J 2005, 4:6I.

29. Liu YT, Old JM, Miles K, Fisher CA, Weatherall DJ, Clegg JB: Rapid detection of alpha-thalassaemia deletions and alpha-globin gene triplication by multiplex polymerase chain reactions. $\mathrm{Br}$ jHaematol 2000, 108:295-299.

30. Carvalho LJM, Oliveira SG, Theisen M, Alves FA, Andrade MCR, Zanini GM, Brigido MCO, Oeuvray C, Povoa MM, Muniz JAPC: Immunization of Saimiri sciureus monkeys with Plasmodium falciparum merozoite surface protein-3 and glutamate-rich protein suggests that protection is related to antibody levels. Scand J Immunol 2004, 59:363-372.

31. Theisen M, Vuust J, Gottschau A, Jepsen S, Hogh B: Antigenicity and Immunogenicity of Recombinant Glutamate-Rich Protein of Plasmodium-Falciparum Expressed in Escherichia-Coli. Clin Diagn Lab Immunol 1995, 2:30-34.

32. Touré FS, Deloron P, Migot-Nabias F: Analysis of Human Antibodies to Erytrhocyte Binding Antigen 175 Peptide 4 of Plasmodium falciparum. Clin Med Res 2006, 4: I-6. 
33. Kocken CHM, Withers-Martinez C, Dubbeld MA, van der Wel A, Hackett F, Blackman MJ, Thomas AW: High-level expression of the malaria blood-stage vaccine candidate Plasmodium falciparum apical membrane antigen I and induction of antibodies that inhibit erythrocyte invasion. Infect Immun 2002, 70:447I-4476.

34. Bonnet S, Petres S, Holm I, Fontaine T, Rosario S, Roth C, Longacre S: Soluble and glyco-lipid modified baculovirus Plasmodium falciparum C-terminal merozoite surface protein I, two forms of a leading malaria vaccine candidate. Vaccine 2006, 24:5997-6008.

35. Jensen ATR, Magistrado P, Sharp S, Joergensen L, Lavstsen T, Chiucciuini A, Salanti A, Vestergaard LS, Lusingu JP, Hermsen R: Plasmodium falciparum associated with severe childhood malaria preferentially expresses PfEMPI encoded by group A var genes. J Exp Med 2004, 199: I 179-1 I 90.

36. Joergensen L, Turner L, Magistrado P, Dahlback MA, Vestergaard LS, Lusingu JP, Lemnge M, Salanti A, Theander TG, Jensen ATR: Limited cross-reactivity among domains of the Plasmodium falciparum clone 3D7 erythrocyte membrane protein I family. Infect Immun 2006, 74:6778-6784.

37. Hogh B, Marbiah NT, Burghaus PA, Andersen PK: Relationship Between Maternally Derived Anti-Plasmodium Falciparum Antibodies and Risk of Infection and Disease in Infants Living in An Area of Liberia, West-Africa, in Which Malaria Is Highly Endemic. Infect Immun 1995, 63:4034-4038.

38. Staalsoe T, Giha HA, Dodoo D, Theander TG, Hviid L: Detection of antibodies to variant antigens on Plasmodium falciparum infected erythrocytes by flow cytometry. Cytometry 1999, 35:329-336.

39. Ronn AM, Msangeni HA, Mhina J, Wernsdorfer WH, Bygbjerg IC: High level of resistance of Plasmodium falciparum to sulfadoxine-pyrimethamine in children in Tanzania. Trans $R$ Soc Trop Med Hyg 1996, 90: 179-I8I.

40. Mutabingwa T, Nzila A, Mberu E, Nduati E, Winstanley P, Hills E, Watkins W: Chlorproguanil-dapsone for treatment of drug-resistant falciparum malaria in Tanzania. Lancet 200I, 358:1218-1223.

4I. Mugittu K, Abdulla S, Falk N, Masanja H, Felger I, Mshinda H, Beck HP, Genton B: Efficacy of sulfadoxine-pyrimethamine in Tanzania after two years as first-line drug for uncomplicated malaria: assessment protocol and implication for treatment policy strategies. Malar J 2005, 4:

42. Nzila AM, Mberu EK, Sulo J, Dayo H, Winstanley PA, Sibley CH, Watkins WM: Towards an understanding of the mechanism of pyrimethamine-sulfadoxine resistance in Plasmodium falciparum : Genotyping of dihydrofolate reductase and dihydropteroate synthase of Kenyan parasites. Antimicrob Agents Chemother 2000, 44:991-996.

43. Holmgren G, Gil JP, Ferreira PM, Veiga MI, Obonyo CO, Bjorkman A: Amodiaquine resistant Plasmodium falciparum malaria in vivo is associated with selection of pfcrt $76 \mathrm{~T}$ and $\mathrm{pfmdrl} 86 \mathrm{Y}$. Infect Genet Evol 2006, 6:309-3 I 4.

44. Soe S, Theisen M, Roussilhon C, Khin SA, Druilhe P: Association between protection, against clinical malaria and antibodies to merozoite surface antigens in an area of hyperendernicity in myanmar: Complementarity between responses to merozoite surface protein 3 and the 220-kilodalton glutamaterich protein. Infect Immun 2004, 72:247-252.

45. Polley SD, Tetteh KKA, Lloyd JM, Akpogheneta OJ, Greenwood BM, Bojang KA, Conway DJ: Plasmodium falciparum merozoite surface protein 3 is a target of allele-specific immunity and alleles are maintained by natural selection. J Infect Dis 2007, 1 95:279-287.

46. Hogh B, Petersen E, Dziegiel M, David K, Hanson A, Borre M, Holm A, Vuust J, Jepsen S: Antibodies to A Recombinant GlutamateRich Plasmodium-Falciparum Protein - Evidence for Protection of Individuals Living in A Holoendemic Area of Liberia. Am J Trop Med Hyg 1992, 46:307-3।3.

47. Dodoo D, Theisen M, Kurtzhals JAL, Akanmori BD, Koram KA, Jepsen S, Nkrumah FK, Theander TG, Hviid L: Naturally acquired antibodies to the glutamate-rich protein are associated with protection against Plasmodium falciparum malaria. J Infect Dis 2000, I 81: I 202-1205.

48. Theisen M, Soe S, Oeuvray C, Thomas AW, Vuust J, Danielsen S, Jepsen S, Druilhe P: The glutamate-rich protein (GLURP) of
Plasmodium falciparum is a target for antibody-dependent monocyte-mediated inhibition of parasite growth in vitro. Infect Immun 1998, 66: I I- I7.
Publish with Biomed Central and every scientist can read your work free of charge

"BioMed Central will be the most significant development for disseminating the results of biomedical research in our lifetime. "

Sir Paul Nurse, Cancer Research UK

Your research papers will be:

- available free of charge to the entire biomedical community

- peer reviewed and published immediately upon acceptance

- cited in PubMed and archived on PubMed Central

- yours - you keep the copyright

Submit your manuscript here:

http://www.biomedcentral.com/info/publishing_adv.asp 